\title{
3. Research on Political Behavior at the Survey Research Center
}

\author{
The SRC has mainly focussed its 1952 and 1956 \\ election studies upon explaining the vote through \\ variables of party identification, issues, and \\ candidate personalities, with some attention to \\ voters' personalities, and somewhat less atten- \\ tion to the intervening layers of variables such \\ as the communication process, economic condi- \\ tions, and voting laws. The 1958 study deals \\ with relations between candidates and voters in \\ their districts.
}

The analytical model that the Survey Research Center has followed in its political research derives from the general ahistorical approach which is associated with the name of Kurt Lewin. As a necessary prelude to focussing our attention on the external conditions of life that define the voter's position in the structure of society, we concentrated upon describing the psychological field as it exists for the voter at the time of his voting decision. Setting aside for the moment the question of how the psychological field has developed through time to its present character, we have sought to identify the major dimensions of this field, to measure them, and to use them to explain the vote.

Our first efforts along this line led us to isolate three major variables, which we identified as party identification, issue orientation and candidate orientation. These we took to represent major motivational forces on the voter, the first expressing the influence of party loyalty, the second reflecting the partisanship of the voter's issue positions, and the third expressing his reaction to the personal qualities of the two presidential candidates. As our 1952 book demonstrates, voters characterized by different patterns of these three forces differ predict- ably and substantially in various attributes of their political behavior.

In 1956 we were able to refine these basic variables into a pattern of six. Because of the open-ended methods of interviewing through which our data are gathered we can now divide the issue variable into partisanship on foreign issues and domestic issues; we represent the reactions to the two presidential candidates as separate variables rather than combined; and we replace the party identification measure by two measures which represent the major attributes of the two parties--their competence as managers of government and their association with the welfare of major social groupings--as they are seen by the voters. (Party identification, as the most important of all group identifications in political behavior, is assigned a separate conceptual role.) When combined in a multi-variate analys is these six "immediate partisan attitudes" have a multiple correlation of .72 with the partisanship of the vote.

When one considers the uncorrected unreliability of our attitudinal measures and the fact that we are working with a dichotomous 
dependent variable, it is clear that further substantial gains at this level in our explanation of preference will be difficult to accomplish. We have been less successful in utilizing our system of attitudinal variables to account for the actual act of voting. We have added to the six partisan variables three "immediate nonpartisan attitudes" which we take to exist in more or less degree in the psychological field at the time of the decision to vote or not to vote. These variables, called sense of political effectiveness, sense of civic duty, and cynicism regarding government, can be shown to exert an influence on the vote turnout which is independent of that expressed in the partisan variables. The fact that all of these variables taken together still do not give a very satisfactory account of the vote is due of course to the presence of a number of factors which are external to this attitudinal system. These include such mundane considerations as the weather, distance from the polls, health, and local restrictions on the franchise, as well as the effects of direct social influence, both facilitating and inhibiting. A full-blown attempt to predict the vote undoubtedly must take these external factors into account.

The fact that we have been relatively successful in representing the voter's motivational field as it relates to his voting preference leads us now to look backward in the sequence of causal events that lies behind the immediate moment of decision. This brings into our analytical map three additional types of variables, those representing the world of external events within which the person lives, those having to do with the process by which these facts are communicated to him, and those which describe those more or less basic attributes of the person himself through which this incoming information is screened and fed into the immediate psychological field.

The process of communication through which the events of the external world are brought to the individual citizen represents an area of investigation that the Center's political program has left virtually untouched. The manner in which the reception of bits of political information is influenced by the intrinsic character of the message, the source from which it originates, the medium through which it travels or the circumstances under which it is received is obviously a research problem of great interest. In its ordering of research priorities the Center has not been able to give such problems more than passing attention.

As will be apparent from the foregoing, we have not concentrated our efforts on finding direct relationships between the facts of the external world and the act of voting without reference to the various layers of intervening variables described above. However, we do have a great deal of interest in the manner in which external events mold the character of the intervening characteristics of the person and are in turn translated by this intervening screen into those elements which make up the immediate psychological field. We are concerned, for example, with the extent to which economic distress affects the intensity of party identification but we also want to know how party identification influences the perception of economic conditions and converts it into positive or negative partisan attitudes. Or we may ask how a political message, once transmitted to a group membership through an official group organ, is received by those members who vary in attachment to the group: How intensely is it 
perceived? Is it perceived as legitimate? Does it have effects on behavior? We are interested, of course, not only in those external events which are contemporary but also those which have occurred in the past but have consequences which persist into the present. Where it is possible to compare data taken at different points in time we have undertaken to show how the political implications of structural variables, for example social class position, are altered by ongoing external events, such as war, post-war readjustment, and subsequent economic prosperity.

Most recently this interest has taken the form of emphasizing some of the more obvious political elements of the institutional context within which the voter must operate. By attending to such things as the extent of partisan competition in the voter's community or the legal boundaries on voting behavior supplied by the election codes, we attempt to further our interest in delineating the interrelationships between individual and "societal" or institutional variables. Broadiy conceived, our program of investigation is one of relating to potentially experienced phenomena of the external world the political attitudinal variables which describe the voter's immediate psychological field to the external world. We view the changes in attitudes over time as, in part, a direct reflection of changes in the national or international political scene. They are also seen as changes which are mediated by the various social structures and processes which define the individual's world--the group memberships, the changes in social and occupational status, the geographical movement of persons from job-to-job and home-tohome--the things that embrace much of the daily routine of living in the American community.
Moving backwards in the presumed causal sequence we concern ourselves with a variety of rather deep-lying personal characteristics that we believe may have an antecedent relationship to the immediate attitudes and to the vote. We divide these into (1) personality traits and (2) what we may call basic orientations, the former being somewhat prior to the latter in causal relationship.

\section{Basic Orientations}

Values. It seems probable that the basic values of security, freedom, humanitarianism, fair play, progress and the like that characterize our culture play an important role in translating the facts of the external world into the attitudes which comprise the immediate psychological field. As yet we have only inferential evidence regarding the political significance of these values; a more detailed investigation of them along the lines suggested by M.B. Smith would be clearly desirable.

Ideologies. Although the American voter is typically not a highly ideological person, some voters clearly are. The role of these more or less firmly structured patterns of belief in determining political attitudes and behavior is a matter of substantial interest in our current series of studies.

Outlook. It has become apparent in the Center's research that the public is subject to rather slow-moving shifts in mood that express a change in optimism or pessimism in outlook. These changes in outlook are undoubtedly effected by external events but they also appear to influence the more immediate attitudes. As intervening variables they claim our attention, in the analysis of economic attitudes and behavior 
as well as political.

Group Identifications. One of the major emphases of our 1956 study was on the political significance of personal identification with the major social groupings in the electorate. This goes beyond the simple structural

placement of the individual in the familiar sociological categories; it involves us rather in questions of the intensity of personal attachment to such social groups as labor unions, farmers, Catholics, $\mathrm{Ne}-$ groes or political parties. We are now concerned with defining the circumstances under which these group identifications may be expected to have greater or less influence on political attitudes and behavior.

\section{Personality Traits}

It is still an open question whether those general attributes of personal style that are commonly called personality traits will be found to account for any significant part of the variance in the political behavior of the general electorate. Our efforts along this line have not as yet achieved very convincing results. However, we do not regard it as unreasonable to expect that political perceptions and attitudes, and consequently behaviors, should be subject to influence by the deeper inclinations of personality and we are at present concerned with the development of measuring instruments that will permit us to pursue this type of inquiry more effectively.
It is not intended in this brief outline of the Center's approach to the analysis of electoral behavior to imply that we have brought all or most of the variables that are included in our map into a coherent and tightlyrelated pattern. In large part our analyses have bridged no more than two adjoining layers of the sequence of variables. We are experimenting with analytical procedures of a more inclusive character, however, and we aspire to a much fuller understanding of the sequence of causal events lying behind the voter's decision than we currently have.

To bring this statement fully up to date we should add that the study of the 1958 congressional elections in which the Center is currently engaged adds a dimension to our program that is not represented in the foregoing description. This study takes us into a comparison of the electorate and the men they elect. Data gathered from both the voters and the candidates who stood for election in their districts will make possible an analysis of the relations between these two levels of participants in the political process that we believe to be unique in research on political behavior in the United States.

--Angus Campbell Survey Research Center University of Michigan

To gain an exact idea of a science one must practice it, and, so to speak, live with it. That is because it does not entirely consist of some propositions which have been definitively proved. Along side of this actual, realized science, there is another, concrete and living, which is in part ignorant of itself, and yet seeks itself; besides acquired results, there are hopes, habits, instincts, needs, presentiments so obscure that they cannot be expressed in words, yet so powerful that they sometimes dominate the whole life of the scholar. All this is still science; it is even its best and largest part, for the discovered truths are a little thing in comparison with those which remain to be discovered.--Durkheim, Of the Division of Labor in Society, 1893. 